\title{
Development and Design of a Zero-G Liquid Quantity Gauge for a Solar Thermal Vehicle
}

\author{
Franklin T. Dodge ${ }^{1}$, Steven T. Green ${ }^{1}$, Steven P. Petullo ${ }^{1}$, and Neil T. Van Dresar ${ }^{2}$ \\ ${ }^{1}$ Southwest Research Institute, San Antonio, Texas 78238 \\ 210-684-5111; fdodge@swri.org; sgreen@swri.org; spetullo@swri.org \\ ${ }^{2}$ NASA-Glenn Research Center, Cleveland, Ohio 44135 \\ 216-977-7533; Neil.T.VanDresar@grc.nasa.gov
}

\begin{abstract}
The development and design of a cryogenic liquid quantity gauge for zero-g applications is described. The gauge, named the Compression Mass Gauge (CMG), operates on the principle of slightly changing the volume of the tank by an oscillating bellows. The resulting pressure change is measured and used to predict the volume of vapor in the tank, from which the volume of liquid is computed. For each gauging instance, pressures are measured for several different bellows frequencies to enable minor real-gas effects to be quantified and thereby to obtain a gauging accuracy of $\pm 1 \%$ of tank volume. Southwest Research Institute ${ }^{\mathrm{TM}}$ and NASA-GRC have developed several previous breadboard and engineering development gauges and tested them in cryogenic hydrogen and nitrogen to establish the gauge capabilities, to resolve several design issues, and to formulate data processing algorithms. The CMG has been selected by NASA's Future X program for a flight demonstration on the USAF/Boeing Solar Thermal Vehicle Space Experiment (SOTVSE). This paper reviews the design trade studies needed to satisfy the SOTVSE limitations on CMG power, volume, and mass, and describes the mechanical design of the CMG.
\end{abstract}

\section{INTRODUCTION}

The problem of accurately gauging the quantity of liquid in a tank in a weightless environment has puzzled spacecraft designers for years. Clearly, earth-based methods such as determining the location of the liquid interface or weighing the tank cannot be employed. Many innovative zero-g gauging methods have therefore been proposed. Several of the methods depend on imaging the shape of the liquid volume (somewhat similar to medical tomography), so they would require substantial instrumentation, long gauging times, and powerful computing resources. For some other methods (e.g., microwave excitation of cavity resonances) it is not evident how the measurements can be interpreted in terms of liquid quantity without calibrating the tank and liquid in weightlessness over a range of fill levels. The straightforward method of metering the outflow from the tank is generally not sufficiently accurate (especially for cryogens). Mord, et al (1988) evaluated many of the proposed zero-g gauging methods and concluded that the compression mass gauge (CMG) method and the pressurevolume-temperature (PVT) method are the two best choices.

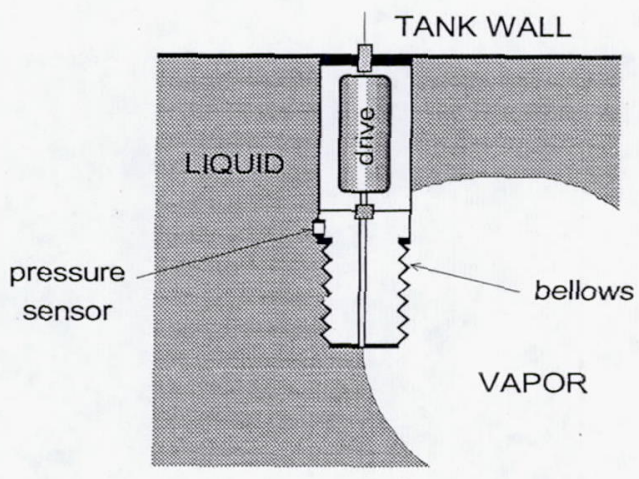

FIGURE 1. Schematic of a Compression Mass Gauge

The CMG method is illustrated schematically in Figure 1. A bellows is used to change the tank volume slightly and the resulting pressure change is measured. The tank gas volume $V_{g}$ can then be inferred from thermodynamics:

$$
V_{g}=-k P(\Delta V / \Delta P)
$$

This is a preprint or reprint of a paper intended for presentation at a conference. Because changes may be made before formal publication, this is made available with the understanding that it will not be cited or reproduced without the permission of the author. 
where $P$ is the mean static pressure of the tank, $\Delta V$ is the change in volume of the tank (equal to the product of the mean bellows cross section area and the bellows stroke), $\Delta P$ is the change in tank pressure, and $k$ is the isentropic gas constant. The liquid volume is the difference between the tank volume and $V_{g}$. Equation (1) assumes that the compression process is adiabatic, the compression is uniform over the entire fluid volume, the liquid is incompressible, and $\Delta V / V_{g}<<1$. (Some of these assumptions can be eliminated when they are not applicable; for example, Eq. (1) can easily include liquid compressibility.) Inasmuch as the CMG's operating principle is not a function of the shape or orientation of the liquid or gas volumes, or of the gauge orientation with respect to the liquid and gas locations in the tank, a CMG works as well in weightlessness as it does normal gravity.

The PVT method is somewhat similar to the CMG method except that the change in tank pressure is produced by injecting a small measured amount of gas into the tank. The CMG appears to be the better choice of the two because, by oscillating the bellows at a known frequency, data processing techniques can be used to eliminate noise in the oscillatory pressure signal (which is quite small and challenging to measure) whereas the PVT pressure signal is a ramp to a new steady state for which the noise cannot easily be removed. Another potential limitation of the PVT method is that, when used with a cryogen, the pressure increase tends to decay back to the initial pressure. The PVT method, and an analogous method in which a small measured pulse of heat is added to the tank fluid and the temperature change measured (from which the mass of fluid can be inferred), are currently used to gauge the storable propellants of certain telecommunications satellites (Ounougha, Jallade, and Pigot, 1998). Since proposed solar thermal vehicles and other advanced reusable launch vehicles use cryogenic propellants, the compression mass gauge is especially relevant for them.

\section{PREVIOUS CMG EXPERIMENTS}

As part of their evaluation of zero-g gauges, Mord, et al (1988) constructed and tested a breadboard CMG in an oilbarrel-size tank containing water and air. The bellows was oscillated at a frequency $f$ of a few hertz, and $\Delta V$ and $\Delta P$ were interpreted as the amplitudes of the corresponding sinusoidal signals. It was found that small non-ideal gas effects prevented the desired $\pm 1 \%$ gauging accuracy from being obtained unless the non-ideal gas effects were accounted for in the data processing. In particular, Eq. (1) had to be modified to account for a non-uniform isentropic gas constant $k$ caused by the temperature change in the viscous boundary layers; to the first order, the relation between the in-phase components of the oscillatory $\Delta V$ and $\Delta P$ signals was found to be:

$$
V_{g}=\frac{k P(\Delta V / \Delta P)}{1+C / \sqrt{f}}
$$

where $C$ is a parameter that depends on fluid properties and liquid-gas-tank configuration. Although $C$ cannot be predicted accurately, Mord, et al (1988) determined it empirically by measuring $\Delta P$ at two frequencies and using Eq. (2) twice to determine both $C$ and $V_{g}$. As expected, the magnitude of $C / \sqrt{ } f$ was found to be much smaller than unity.

Monti and Berry (1994) tested a breadboard-like CMG in a Get Away Special experiment during the flight of STS57 in 1993. They used Eq. (1) to analyze gauging data for a small tank containing Fluorinert ${ }^{\mathrm{TM}}$ FC75. Good accuracy was found. The value of $\Delta V / V_{g}$ used in these tests was, however, relatively large (compared to what would be practical for a space vehicle) so as to enable $\Delta P$ to be more easily measured.

Rogers, Dodge, and Behring (1995) used a breadboard CMG to investigate the effects of cryogenic liquids. Their tests employed an oil-barrel-size tank containing saturated liquid and vapor Freon ${ }^{\mathrm{TM}}$, to simulate a cryogenic propellant. The tests revealed that mass transfer of the near-boiling fluid at the liquid-vapor interface had a noticeable influence on gauging accuracy. During each bellows oscillation cycle, vapor tended to condense when the tank pressure increased and liquid tended to vaporize when the tank pressure decreased; in both cases, the pressure change was less than that predicted by the ideal gas law. Although mass transfer effects can be diminished by using a sufficiently high bellows frequency, there is a limit on the highest frequency that can be employed without introducing other unwanted effects, as will be discussed later. By analyzing the heat transfer rate at the liquid-vapor interfaces, Saiyed (1993) predicted that the mass transfer effect could be treated by a further refinement of Eq. (2):

$$
V_{g}=\frac{k P}{1+C / \sqrt{f}}\left(\frac{\Delta V}{\Delta P}-\frac{K}{\sqrt{f}}\right)
$$


where $K$ is an empirical "mass-transfer" coefficient. Values for $K, C$, and $V_{g}$ were established by using three or more distinct bellows frequencies for each gauging instance. The values of both $K / \sqrt{ } f$ and $C / \sqrt{ } f$ were found to be small but crucial in obtaining good gauging accuracy.

\section{CRYOGENIC TESTING OF THE ENGINEERING DEVELOPMENT MODEL CMG}

Because of the applicability of a CMG to cryogenic propellants, Southwest Research Institute ${ }^{\mathrm{TM}}$ and NASA-Glenn Research Center entered into a Space Act Agreement in the early 1990s to develop the CMG technology to the point where it could be realistically tested in a space experiment. To that end, an Engineering Development Model CMG was fabricated that was suitable for service in cryogenic hydrogen or nitrogen and of a size that was compatible with an existing $0.167 \mathrm{~m}^{3}\left(5.9 \mathrm{ft}^{3}\right)$ dewar at NASA-GRC. Since the unit was meant only for development purposes, no attempt was made to minimize its size, weight, or power as would be required for a vehicle application. Figure 2 shows an exploded photograph of the CMG. The overall volume of the CMG was about $0.012 \mathrm{~m}^{3}\left(0.42 \mathrm{ft}^{3}\right)$. The bellows was of a welded stainless steel design and had a mean diameter of $64 \mathrm{~mm}$ (2.5 in) and a zero-to-peak stroke of about $6.2 \mathrm{~mm}(0.25 \mathrm{in}$ ), which produced a $\Delta V$ (zero-to-peak) of $0.012 \%$ of the dewar free volume; this $\Delta V$ is a representative value for an actual space vehicle application. An electric motor was used to drive the bellows.

A number of development problems had to be solved during the CMG design and testing. For example, oscillatory pressures in the range of $100 \mathrm{~Pa}(0.015 \mathrm{psi})$ had to be measured for frequencies as low as $3 \mathrm{~Hz}$. Piezoelectic transducers for cryogenic service are commercially available with the required sensitivity, but developing a calibration curve for them at such low frequencies and low amplitudes was a challenge (Green, 1998).

A least-squared error algorithm was used to analyze the gauging measurements. Customary least-squared-error methods could not, however, be applied since the desired quantity $V_{g}$ is not known. After examining several alternatives, a method based on fitting a pressure parameter to the analytical model was developed from Eq. (3):

$$
\sum E_{i}^{2}=\left[\left(\frac{k P \Delta V}{\Delta P}\right)_{i}-V_{g}-\frac{V_{g} C+k P K}{\sqrt{f_{i}}}\right]^{2}
$$

where $E_{i}$ is the error. By considering $\left(V_{g} C+k P K\right)$ and $V_{g}$ as the two parameters to be determined and $1 / \sqrt{ } f$ as the variable, a set of linear equations was derived that could be easily solved for the best or "true" value of $V_{g}$ (Dodge and Kuhl, 1997). The experimental $\Delta P_{i}$ amplitude data were computed from the digitized pressure time-history signal for each bellows frequency $f_{i}$. Low frequency drift and high frequency noise were eliminated from the $\Delta P$ measurements by a combination of Fast Fourier Transform, spectral power content analysis, and a process that located the part of each $\Delta P$ record that best eliminated spectral leakage.

An extensive series of tests was conducted with liquid hydrogen and nitrogen for a variety of tank fili leveîs and pressures (Jurns and Rogers, 1995; Dodge and Kuhl, 1997, 1998); for some tests, helium gas was used to pressurize the tank above the fluid saturation pressure. To establish the liquid volume independently of the CMG measurements, the cryogenic dewar was instrumented with an array of vertically-oriented silicon diode sensors (which measure temperature) to determine the approximate liquid level during filling, and the fluids in the dewar were weighed by a load cell

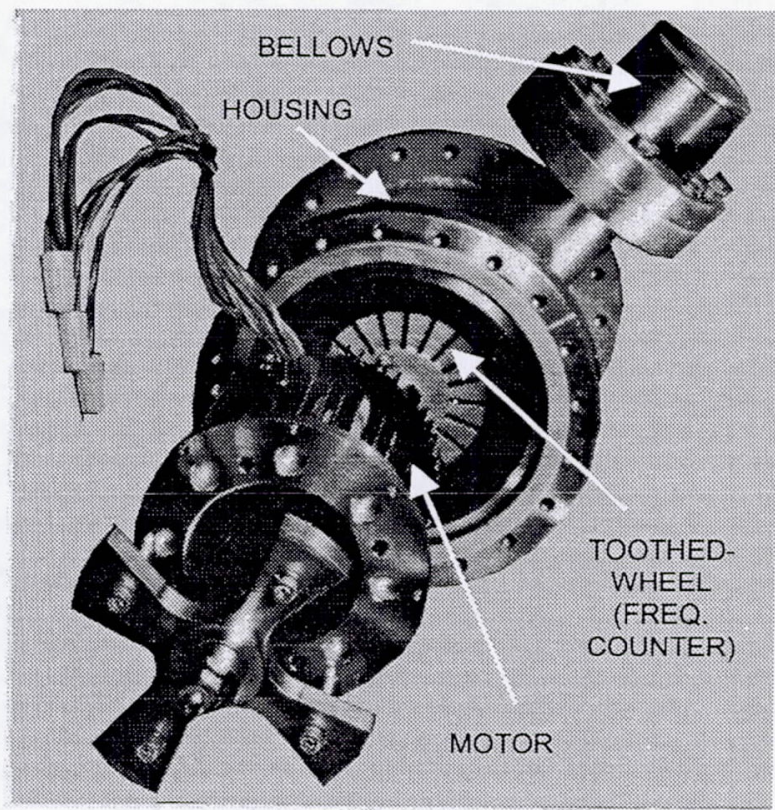

FIGURE 2. CMG Engineering Development Model 
during each test. Nonetheless, because of heat leaks and other problems, there were questions about the accuracy of these determinations. In addition to the uncertainty introduced by the dewar fluid measurement system, the mechanical operation of the CMG also deteriorated such that the actual $\Delta V$ varied slightly in an unknown way during the later tests. These problems limited the degree to which the accuracy of the CMG could be established. (The CMG has since been upgraded and refurbished, and the NASA-GRC facility has been greatly improved.)

Figure 3 shows representative results, in this case for liquid nitrogen, from tests conducted over a period of months. Some of the tests conducted near the end of the testing are marked as "questionable" in the plots, for the reasons noted above. The averages of the tests considered to be valid are also indicated in the plots. In general, the averages predicted the vapor volume to within $\pm 2 \%$, which is considered satisfactory since there was probably at least $\pm 1 \%$ error in the vapor volume determined by the dewar load cell used for comparison to the $\mathrm{CMG}$ results.

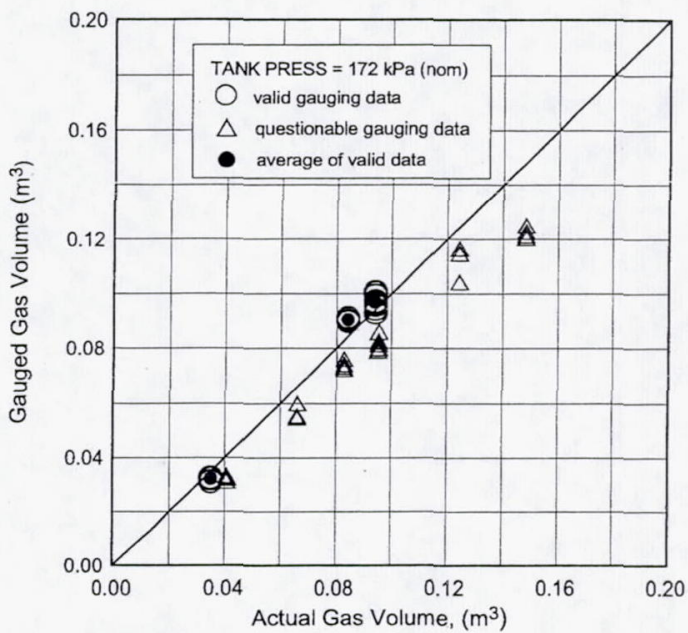

FIGURE 3. Gauging results for LN2 at a tank pressure of $172 \mathrm{kPa}$ (nominal)

\section{DESIGN OF A CMG FOR A SOLAR THERMAL VEHICLE}

Because of the CMG capabilities demonstrated by the cryogenic ground tests, a flight experiment was proposed to and accepted by NASA's Future X program. The vehicle selected for the experiment was the USAF/Boeing Solar Thermal Vehicle Space Experiment (SOTVSE) which employs liquid hydrogen as its propellant. The $\mathrm{LH}_{2} \operatorname{tank}$ volume is about $1.89 \mathrm{~m}^{3}\left(66.7 \mathrm{ft}^{3}\right)$. For the baseline SOTVSE design, the tank pressure at launch is about $124 \mathrm{kPa}$ (18 psia) and the liquid fill percentage is about $89 \%$. After reaching low-earth orbit, the tank remains in a locked-up configuration for several days, while the propellant is heated to raise the tank pressure to about $310 \mathrm{kPa}(45 \mathrm{psia})$, at which point the fill percentage has increased to $96 \%$ as a result of expansion of the $\mathrm{LH}_{2}$. Thrusters fed from the tank are then activated during each orbit to raise the orbit until the $\mathrm{LH}_{2}$ is exhausted. SOTVSE is an almost ideal vehicle to demonstrate the $\mathrm{CMG}$ technology, for the following reasons:

- The fill level changes from $89 \%$ to $96 \%$ with no change in liquid mass; thus the CMG accuracy can be verified over a range of fill levels for conditions known with good accuracy.

- The tank contains an array of heated silicon diode sensors from which the settled liquid level (measured during thrusting) can be determined at a number of discrete locations; these measurements also allow the $\mathrm{CMG}$ accuracy to be determined for a variety of fill levels.

- The $\mathrm{LH}_{2}$ is continually mixed to prevent stratification.

- The LH2 is stored in a saturated condition without a non-condensable pressurizing gas.

SOTVSE does, however, impose limitations on the allowed CMG power, volume, and weight. In addition, the fairly large change in tank pressure from launch to operation and the fact that the tank is "upside down" at launch presented a challenge to the design of the gauge.

\section{Objectives of the CMG Space Experiment}

The overall objective of the space experiment is to demonstrate the gauging and operational capability of the CMG in an actual space vehicle and thus, in NASA's terminology, to achieve a Technology Readiness Level of 7. Specifically, the CMG design will incorporate all the features needed to make it flight-like, and the experiment will investigate, to the extent possible, all the relevant zero-g physical effects that might influence the operation of a compression mass gauge in a space environment, especially those that cannot be reasonably simulated by ground tests. These zero-g effects, listed in the likely order of their importance, are:

- location and configuration of liquid and vapor zones 
- resonances between multiple vapor bubbles produced by the bellows motion

- liquid sloshing created by the bellows motion

- cavitation at the gauge head induced by the failure of liquid to follow the bellows motion

- thermal stratification

Since the extent of these effects cannot be predicted in the absence of low-g test data, the flight gauge will be designed with enough "intelligence" to recognize the effect and compensate for it if necessary. For example, the software will filter out a cavitation pressure spike. Similarly, if the magnitude of $\Delta P$ changes significantly when $f$ changes, which would thus indicate the occurrence of a multiple bubble resonance, the software will change the bellows oscillation frequency range.

\section{Trade Studies}

The SOTVSE design and operation puts limits on the CMG power, mass, and volume; these limits are roughly 75 watts peak power and then only for brief durations, total mass of $45 \mathrm{~kg}$ (100 lbs), and total volume of $1 \%$ of tank volume. Trade studies were therefore conducted to develop the flight CMG hardware design such that the tank liquid volume can be gauged over a representative fill level range with the desired accuracy of $\pm 1 \%$ of tank volume.

Power was one of the more important trade studies. The resolution of the $\Delta P$ sensor is finite, so the theoretically-best resolution of the CMG is also finite. Equation (1) can be used to show that, for a fixed, finite $\Delta P$ resolution, the $\Delta V$ required to obtain a given gauging accuracy increases dramatically for low fill levels. Since, with other things held constant, the power to drive the bellows increases with $\Delta V$, it is concluded that, for a given power budget, there is a lower limit on the tank fill level that can be gauged with the desired accuracy. The required power also depends on the bellows frequency $f$, the bellows spring stiffness, the internal volume of the gauge enclosure (since the internal gas is compressed as the bellows oscillates), the vapor volume of the tank, the pressure differential between the gauge internal volume and the tank, and the fluid properties. An analytical model was developed to relate these parameters to the electric motor power. The enclosed volume of the CMG was found to be an important parameter since the gas contained in the volume acts like a pneumatic spring during the bellows oscillation. The pressure differential between the CMG enclosed volume and the SOTVSE tank was also found to quite important; for that reason, the design includes a pressure equalization scheme to maintain the pressure differential at near zero.

These trade studies indicated that, to satisfy the CMG power, weight, and volume budgets, the gauged fill levels had to be greater than about $65 \%$ to meet the desired accuracy goal of $\pm 1 \%$ of tank volume. It is expected that, after the space experiment data are analyzed, less conservative design approaches will be feasible.

\section{Pressure Equalization Design}

The SOTVSE CMG is nominally a completely sealed enclosure. It will be purged of air and filled with gaseous helium to one atmosphere before being installed in the SOTVSE tank. When the SOTVSE tank is being loaded with $\mathrm{LH}_{2}$ prior to launch, the CMG temperature and gas pressure will decrease substantially, and a large pressure differential would therefore be created between the tank and the CMG if the CMG remained sealed. Since this pressure differential is large enough to overstress the bellows, the pressure differential must be prevented. As mentioned previously, a pressure equalization method is also needed during flight (when the SOTVSE tank pressure changes) to minimize the power required to oscillate the bellows.

After numerous concepts were analyzed, an equalization method was chosen that employs vaporization and condensation of cryogenic hydrogen. A solenoid valve is incorporated in the CMG housing to ailow the CMG internal volume to communicate with the SOTVSE tank volume. During the tank filling prior to launch, liquid level sensors will open the valve when $\mathrm{LH}_{2}$ reaches a location just above the bottom of the CMG; this ensures that the CMG hardware temperature is the same as the $\mathrm{LH}_{2}$ temperature and that the tank has been sufficiently chilled so that only hydrogen vapor will flow across the solenoid valve into the CMG. The vapor flow into the CMG will keep the $\mathrm{CMG}$ internal and tank pressure equalized. The $\mathrm{CMG}$ also incorporates a small internal standpipe connected to the solenoid valve. At some point during the filling, the valve inlet will become submerged in liquid, and liquid will flow from the tank through the valve into the CMG standpipe. At that point, both the tank and the CMG pressure will be very close to the final tank pressure. After a small amount of liquid has flowed into the standpoint to fill it to a pre-determined level, the solenoid valve will close. The internal and tank pressures will remain equalized during the remainder of the tank loading. 
When SOTVSE reaches its initial orbit, the propellant will be heated over a number of days to raise the tank pressure to $310 \mathrm{kPa}$ (45 psia). The CMG temperature will increase in lock-step with the tank, and the $\mathrm{LH}_{2}$ in the CMG standpipe will consequently evaporate into the internal volume to maintain the CMG pressure equal to the SOTVSE tank pressure. During any subsequent gauging operation when the tank pressure may differ somewhat from the nominal $310 \mathrm{kPa}$ pressure, the solenoid valve will open to admit fluid (liquid or vapor) or expel vapor to maintain a zero pressure differential; the amount of fluid exchange needed will be extremely small since the saturated liquid in the CMG standpipe will evaporate or condense in response to the SOTVSE tank temperature/pressure change to maintain pressure equalization. The solenoid valve will be closed just before the actual gauging process.

Although this equalization process may appear to be unduly complicated, it is necessary to ensure that the SOTVSE CMG is self contained and can equalize the pressure differential regardless of whether the external fluid in contact with the CMG is vapor or liquid. For some applications, it may be sufficient merely to plumb the CMG to the tank gaseous pressurization supply to maintain the $\mathrm{CMG}$ internal pressure equal to the tank pressure.

\section{SOTVSE Hardware Design}

Figure 4 illustrates the SOTVSE CMG design. Two identical but opposed bellows are employed to minimize the net oscillatory force and torque exerted on the SOTVSE during the gauging. Both bellows are driven in phase by the same motor through a scotch yoke mechanism. Each bellows has an outside diameter of $126 \mathrm{~mm}$ (4.97 in), and the length from the end of one bellows to the end of the other is $427 \mathrm{~mm}(16.8 \mathrm{in})$. The overall axial length of the CMG is $401 \mathrm{~mm}(15.8 \mathrm{in})$. The cylindrical barrel that houses the electric motor and standpipe has a diameter of $335 \mathrm{~mm}$ (13.2 in) and a length of about $254 \mathrm{~mm}(10 \mathrm{in})$; the volume of this barrel was made as large as possible to minimize the effect of the hydraulic spring of the contained gas on the power requirements. Each bellows is also attached to an inertial mass (reciprocating "flywheel") to help smooth out the load on the electric motor.

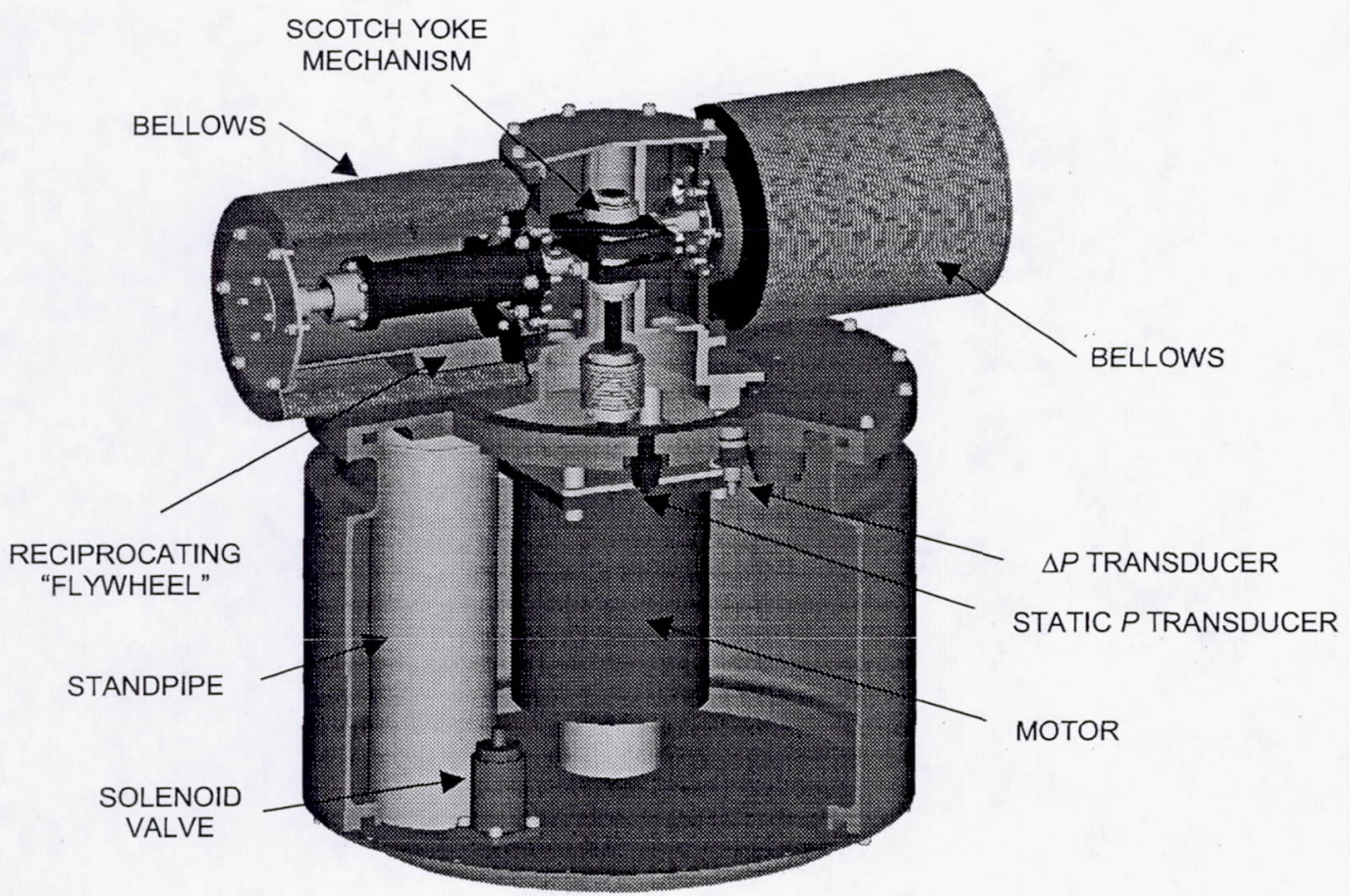

FIGURE 4. Solid model of the SOTVSE CMG 
All the components of the CMG are commercially available and are compatible with cryogenic hydrogen. The bellows are welded stainless steel. For liquid oxygen service, the bellows may need to be of the convolute-formed type; however, convolute-formed bellows generally have a considerably larger spring constant than the welded type, which is the reason the welded type was chosen for the SOTVSE CMG.

\section{COMPENSATION FOR ACOUSTIC EFFECTS IN VERY LARGE TANKS}

For the reasons discussed earlier, the bellows must be operated at three or more frequencies during each gauging instance to determine $V_{g}$. Furthermore, it is greatly beneficial to separate the frequencies as widely as possible, since this reduces the uncertainty in the data analysis procedure. The $\Delta P$ dynamic pressure sensor, however, imposes a lower limit of about $3 \mathrm{~Hz}$ on the allowed bellows frequency, and the volume of the tank imposes an upper limit: if the frequency is too high compared to the acoustic resonance frequency of the gas volume, the acoustic wave travel time through the gas becomes comparable to the bellows oscillation period, and compression is not achieved uniformly throughout the tank. The fundamental CMG principle, Eq. (1) or Eq. (3), is not applicable when the compression is not uniform. The allowed frequency range must fit between these two limits.

A relatively simple order-of-magnitude analytical model of the acoustics effects was used to determine the allowed frequency range of the SOTVSE gauge. The lowest acoustic wave frequency of the SOTVSE tank was estimated to be about $120 \mathrm{~Hz}$. The analytical model showed that, to maintain acoustic effects below the $1 \%$ level, the maximum allowed value of $f$ could not be higher than $12 \mathrm{~Hz}$. The SOTVSE CM bellows frequency range was therefore selected to be $3 \mathrm{~Hz}$ to $12 \mathrm{~Hz}$.

For tanks substantially larger than the SOTVSE tank, the spread between the lowest $(3 \mathrm{~Hz})$ and highest allowed frequencies as determined from the acoustic wave travel time will be too narrow for accurate gauging (Dodge, 2000). The mode of operation would either have to be changed (for example, to a bellows step or "pulse" motion followed by a delay to let the acoustic ringing to decay) or the data analysis would have to account for acoustics effects. Acoustics effects can be approximately accounted for in the analysis by a first order correction to Eq. (3):

$$
V_{g}=\frac{k P}{1+\left(C / \sqrt{f}+D f^{2}\right)}\left(\frac{\Delta V}{\Delta P}-\frac{K}{\sqrt{f}}\right)
$$

where the empirical "acoustics coefficient" $D$ can be determined just as $C$ and $K$ are, although additional $\Delta P$ vs. $f$ measurements would be required. By using Eq. (5) to analyze the gauging data, the bellows frequency can be as high as about one-third the acoustics frequency. Since a reasonably good bellows frequency range is $3 \mathrm{~Hz}$ to $12 \mathrm{~Hz}$, the tank volume could therefore be as large as about $28 \mathrm{~m}^{3}\left(1000 \mathrm{ft}^{3}\right)$, inasmuch as the acoustics resonant frequency would be no smaller than $35 \mathrm{~Hz}$. But for tanks larger than about $28 \mathrm{~m}^{3}$, which could have acoustic resonant frequencies below $35 \mathrm{~Hz}$, the bellows frequency would have to be less than $12 \mathrm{~Hz}$, because, when the bellows frequency is greater than one-third the acoustic resonant frequency, the first order correction employed in Eq. (5) is not sufficiently accurate to represent the acoustic effects. Hence, the allowed frequency range could be so narrow for these very large tanks that a pulse mode of bellows operation might be necessary.

\section{CONCLUSIONS}

The compression mass gauge has been the subject of a ten-year development process between Southwest Research Institute ${ }^{\mathrm{TM}}$ and the NASA-Glenn Research Center. Breadboard and engineering development models of the gauge have been tested with cryogens in tanks of various sizes to establish the important parameters, resolve design issues, and validate data analysis algorithms. This development has shown that a compression mass gauge is able to achieve an accuracy of $\pm 1 \%$ of tank volume for cryogens. The size and power requirements of the gauge also appear to be practical for space vehicles.

The compression mass gauge has the great advantage, compared to most other zero-g gauging methods, that it does not have to be calibrated in the tank in zero-g as a function of fill level, tank shape, and liquid-vapor configuration. The only "calibration" that is required is due to relatively minor real-gas effects, not by complicated tank shapes or liquid-vapor configurations. This "calibration" to accommodate real gas effects can be easily accomplished during each gauging instance by operating the gauge at several bellows frequencies. 
As a result of the previous developments, the compression mass gauge technology has been selected by NASA's Future X program for a flight demonstration on the USAF/Boeing Solar Transfer Vehicle Space Experiment. The SOTVSE propellant is liquid hydrogen. The SOTVSE CMG will be manufactured from flight-qualified components to aid in demonstrating a NASA Technology Readiness Level of 7. The mechanical design of the CMG is complete, although the electronics design has been placed on hold until the SOTVSE electronics interfaces are defined more completely.

At this time, the flight date of the SOTVSE has been postponed for several years. As an interim measure, a smaller version of the SOTVSE CMG is planned, which will be fabricated from flight-qualified components. This CMG, will be suitable for additional ground testing at NASA-GRC and NASA-MSFC. When the SOTVSE development is resumed, the smaller CMG will be ready for installation in the SOTVSE tank. But because of its smaller size, and in particular as a result of the smaller swept volume $\Delta V$ of the bellows, the desired gauging accuracy of $\pm 1 \%$ of $\operatorname{tank}$ volume will be achievable only for SOTVSE tank fill levels greater than about $85 \%$.

\section{NOMENCLATURE}

$C=$ empirical parameter related to viscous boundary layer temperature change $\left(\mathrm{Hz}^{0.5}\right)$

$D=$ empirical parameter related to pressure magnification by acoustic resonances $\left(\mathrm{Hz}^{-2}\right)$

$f=$ bellows frequency $(\mathrm{Hz})$

$k=$ isentropic gas constant

$K=$ empirical parameter related to mass transfer at the liquid-vapor interface $\left(\mathrm{Hz}^{0.5}\right)$

$P=$ mean static pressure of vapor in tank $(\mathrm{Pa})$

$V_{g}=$ volume of vapor in tank $\left(\mathrm{m}^{3}\right)$

$\Delta V=$ amplitude of swept volume of the CMG bellows ( $\mathrm{m}^{3}$, zero-to-peak)

$\Delta P=$ amplitude of oscillating fluid pressure ( $\mathrm{Pa}$, zero-to-peak)

\section{REFERENCES}

Dodge, F. T., and Kuhl, C. A., "Analytical Models and Data Analysis Methods for the Smart Compression Mass Gauge Upgrade," Southwest Research Institute, Final Report, IR\&D Project 04-9014, San Antonio, Texas (1997).

Dodge, F. T., and Kuhl, C. A., " $\mathrm{LN}_{2}$ Gauging Tests Using the Smart Cryogenic CMG," Southwest Research Institute, Final Report, Project 04-8862, NASA-GRC Contract, San Antonio, Texas (1998).

Dodge, F. T., "Compression Mass Gauge for Very Large Cryogenic Tanks," Southwest Research Institute, Final Report, IR\&D Project 18-R9181, San Antonio, Texas (2000).

Green, S. T., "Pressure Sensor Risk Mitigation Study for the Compression Mass Gauge Conceptual Design" Southwest Research Institute, Technical Report, Project 04-1142, NASA Contract C-78267-F, San Antonio, Texas (1998).

Jurns, J. M., and Rogers, A. C., "Compression Mass Gauge Testing in a Liquid Hydrogen Dewar," NASA Contractor Report 198366 (1995).

Monti, R., and Berry, W., "Liquid Gauging in Space: The G-22 Experiment," ESA Journal, 18, 51-61 (1994).

Mord, A. J., Snyder, H. A., Kilpatrick, K. A., Hermanson, L. A., Hopkins, R. A., and Van Gundy, D. A., "Fluid Quantity Gauging," Ball Aerospace Systems Group, Report DRL-5 DRD MA-183T F88-06, NASA Contract NAS9-17616, Boulder, Colorado (1988).

Ounougha, L., Jallade, S., and Pigot, S., "Propellant Gauging Method Applied on MMS Telecommunication Satellite," AIAA Paper 98-3167, $34^{\text {th }}$ AIAA/ASME/SAE/ASEE Joint Propulsion Conference and Exhibit (1998).

Rogers, A. C., Dodge, F. T., and Behring, K. A., "Feasibility Development of a Cryo Fluid Gauging System for Space Vehicle Applications," AIAA J. Spacecraft and Rockets, 11, 980-985 (1995).

Saiyed, N. V.-H, “Applicability of Compression Mass Gauging to Condensible Fluids,” M. Sc. Thesis, Department of Mechanical and Aerospace Engineering, Case Western Reserve University, Cleveland, Ohio (1993). 